\title{
A Survey about Applications, Issues and Challenges of Sensor Network
}

\author{
Dania Farooq \\ Department of Computer Science \\ The University of Lahore \\ Sargodha, Pakistan
}

\author{
Muqaddas Gull \\ Department of Computer Science \\ The University of Lahore \\ Sargodha, Pakistan
}

\begin{abstract}
This research work defines the concept of Sensor network that is consist on smallest nodes that performed some process, collect information of sensing, with calculation, and wireless communications competencies. This concept was taken from micro electro-mechanical systems technology. It consist on standard, application, energy, and techniques. Sensor network routing protocol are design according to the network and architecture. Sensor network needs application domain specialists, hardware inventers, and software designers to present effective systems. By applying sensor network characteristic like, fault tolerance, flexibility, high sensing reliability, less budget, that can generate new application areas for remote sensing devices. Sensor network constraint are need to be analyze so that it can satisfy the knowledge about sensor network these contraint followed by error acceptance, cost, hardware, topologies, and power consumption. It's also very helpful in security, and energy. This research paper shows the overview of the sensor network and its, application with their issues and challenges.
\end{abstract}

\section{Keywords}

Applications, Attributes, Sensor Network, Protocol, Standards.

\section{INTRODUCTION}

Sensor network have the capability to change our lives, by developing different applications with theirs challenges. Wireless Sensor network [1] is the part of ElectroMechanical system which provides development of low cast and power to perform multiple functions of sensor nodes that are small size to create communication component, data processing and these nodes cooperate with each other. Data for small and different environment is obtained from the sensor network and different sensors are at different locations. They also solve some optimization problem which consists of location, deployment and tracking, these are considered as fundamental issues Sensor network provide quality of services like surveillance. Different applications are used in a sensor network that helps in different areas like military applications along with instruction detection, parameters monitoring, health observer, and location revealing and programmer detection.

\section{STANDARDS}

To work with standard organization to help best and efficient solution. There can be two types of standards of wireless sensor network, one group increase data rate throughput and quality of service while the other is decrees data rate, cost [2], create connectivity with other network facilitates, low-volume that does not defend a proper explanation of wirelessly connected devices. IEEE P1451.5 describes Network Capable Application Processor, also, provide a physical interface for Wireless standards like (802.11) are used for Wi-Fi, (802.15.1) for Bluetooth, (802.15.4) for ZigBee.

\section{PROTOCOLS}

In a wireless sensor network, MAC protocols and ISF protocols are introduced. ISF is the most favorable protocol used for the routing purpose in sensornetwork. ISF rules apply hop calculation, position, and data to attain energy productivity in data delivery device. Security Sensor network provides the security by using their constraints like, belief on sensor network to take over important missions such as military required. Sensor network and its topologies come with various threats due to the vulnerability of the topologies in the network. It defines the detail of clustered, unclustered which are network topologies undergoing models of risk such as node disasters, and multiple attacks. The result of cluster topology shows highest level of resistance to perform random attacks, as well as uncluttered is not performed this work. Sensor node is scattered in sensor field. These scattered nodes have abilities to collect data and then route that data back to the sink and end users. Data rout back to the end user by using multi hop infrastructure through the sink as shown in Fig.1. Sink use Internet or Satellite for communicate with task manager. Sink use protocol stack with sensor network nodes that are shown in Figure 1. The protocol stack combined control and routing responsiveness, and increase communication control efficiency by using wireless sensor medium, and promote the efficiency of sensor nodes. Stack protocol contains application, transport, network, data link and physical layers which are shown in Figure 2.

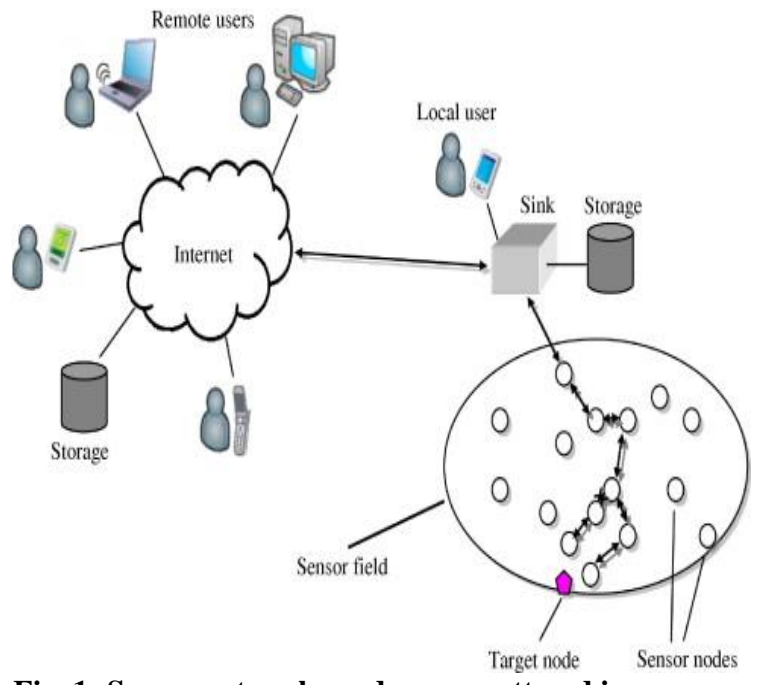

Fig. 1: Sensor networks nodes are scattered in a sensor field 


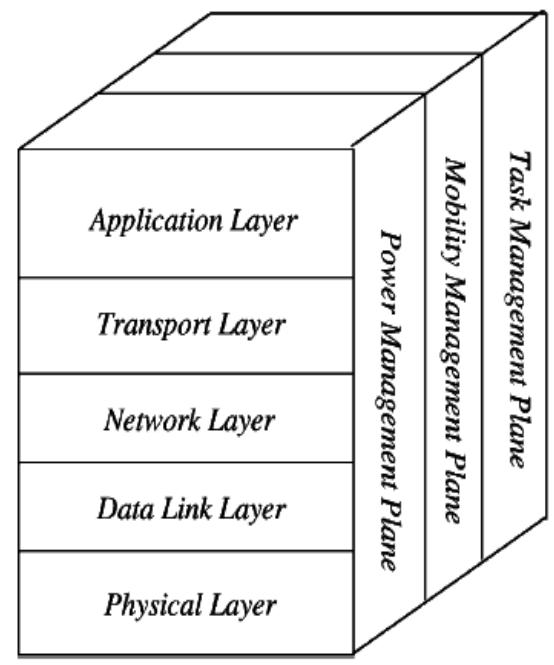

Fig. 2: Sensor network protocol stack

\section{SECURITY}

Security mechanism in a network is very necessary to achieve for secure communication to prevent network from unauthorized access, attacks and hacking the information. Sensor network is consider as to play the best roll in a network due to its memory, computation and power resources. Sensor network considers to take over important missions in the military. In ad-hock network sensor network do not provide the guarantee for reliable security to perform network functionality. Even this mechanism can break down the physical infrastructure. For security reason, the IEEE standard like 802.15.4 [3] gives three levels of security like:-

- A number of security with different types applies in advertising kiosk applications and access control list.

- Security in non-cryptography

- Symmetric key, like advance encryption standard.

\section{MANUFATURE}

Electro-mechanical systems (MEMS) produce the small sensor network about a cubic inch in size, to make able assemble to real-world huge in networks. It can help to solve more complicated task such as signal processing, compression methods, and data collection. The most common tiny sensor device is Motes which is manufactured by Crossbow Inc, and established at UC Berkeley. Motes are used in different data collection applications to perform highly practical and now used for different purpose in environmental monitoring.

Table 1. Different applications and Motes

\begin{tabular}{|c|l|}
\hline $\begin{array}{c}\text { Different } \\
\text { Applications }\end{array}$ & \multicolumn{1}{c|}{ Motes, Insolent Sprinkle Sensors } \\
\hline General form & $\begin{array}{l}\text { Health and Wellness Monitoring, Power } \\
\text { Monitoring, Inventory Location Awareness. } \\
\text { Indoor and Outside Environmental Monitoring, } \\
\text { Security and Tracing. }\end{array}$ \\
\hline Test, & $\begin{array}{l}\text { It contains Accelerometers, Vibration Sensors, } \\
\text { Direction Sensors, Hypnotic Sensors, Inertial }\end{array}$ \\
\hline
\end{tabular}

\begin{tabular}{|c|l|}
\hline Measurement & Sensors \\
\hline $\begin{array}{c}\text { Advance } \\
\text { wireless }\end{array}$ & $\begin{array}{l}\text { Trifling scope, slight rate, unassuming, un } \\
\text { attended, wireless sensor, aboard processing } \\
\text { communications, and dynamic reprogramming }\end{array}$ \\
\hline $\begin{array}{c}\text { Existing } \\
\text { sensor boards }\end{array}$ & $\begin{array}{l}\text { Light and Heat, Magnetometer, Weather } \\
\text { Monitoring }\end{array}$ \\
\hline
\end{tabular}

\section{APPLICATIONS}

Sensor network has initiated the technique [4] in an inclusive variety of bids and systems with varying desires and features. There are different types of application [5] that can work with sensor network like in Industrial, Military Location, Public Safety, Automotive, Airports, Agriculture, Emergency situations, Rotating, Machinery, Medical/Health, Ocean, Home applications, Environmental applications , Commercial applications.

\section{ISSUES IN APPLICATIONS}

In sensor network, there are some issues in applications which are [6].

\subsection{Research Issues in Industrial Applications}

Some issues are occurring in Industriel applications which are developed by distributed architecture to require for inexpensive, flexible and dependable. Sensors and actuators can create direct connection with communication to increase the performance of the system and data can be easily shared on the web. The details of industrial applications .Some industrial applications [7] are based on some technology which performs different functions in the industry they are these The ZigBee technology are used in petrochemical industry that is the part of sensor network applications. Coal mines technology has been describing [8] for safety monitoring. The automatic Meter Reading system is presented for remote online. For telecommunication latest technology are developed like wireless RF, with ZigBee modules [9] and Code Division Multiple Access. WSN based remote meters to provide individuals monitor water, gas and other resources, because the direct access in the physical reading of meters of electricity, gas, and water is not possible so sensor network provides remote meter. Metal cutting process in sensor network used for detection in development. Dynamic Power Management has been defined in sensor network for an electric power system for condition monitoring, diagnosis, and supervisory control. DPM was implemented by sensor nodes to enhance its life time. Human motion sensors are designed to provide security for the power plant. Acoustic sensors are developed for underwater and can be used in deep sea and deep water area.

\subsection{Research Issues in Health Care}

Sensor network working is used in all type of applications and its efficiency is increasing day by day. It also works in medical field to improve the performance of medical applications and make the parameter to do the best performance so that to provide facilities to the patients and deceases can be easily diagnose. physicians and caretakers can monitor remotely the Physiological parameters of patients without effecting or damage the patient's activities, the result shows the improvement of equipment, reduction of cost and 
efficient management of patient gaining viable benefits. These technologies allowed better understanding into the origin of diseases have expressively reduced human errors, and help in developing methods for rehabilitations, recovery, and impact of drug therapy. The main research issue must be focused on the incremental level of awareness in the different medical center like a home assistant, caregivers, and primary healthcare centers, so they quickly understand the health activity status of the patient, distinguish and decide the required action in that situation. It is very useful to create awareness in the people what they should do in the emergency situation and they should know how to use medical equipment in an emergency situation. Received Signal Strength Indicator (RSSI) algorithm was presented for work fine in a home environment and it was proved experimentally and then apply on home applications. Wireless sensor network introduced simple and low-cost healthcare monitor for elder people, where perceptions and acceptance were done for home applications. Sensor network has been developed the technology health care system for the elder patient for home [10] without disturbing their routines. The main issue is to be concerned to develop and monitor the system with, reliability, bandwidth and efficient power. It is necessary to focus on the standardization of display and interoperability in health care system. There is more to understand the specific limitations along with new applications that can be resolute by the study of different medical conditions in clinics.

It should be measured Electro Cardio Gram and acceleration Signals are continuous monitoring with real-time systems. Y.D.Lee and W. Y. Chung developed a small shirt for measurement that is made by conductive fabrics to obtain the body signal as electrodes and they consist with a sensor for online health monitoring. Practically proved that data has been measured and transmitted to the ad-hoc network for remote monitoring. The remote monitoring can be shown in Figure 3.

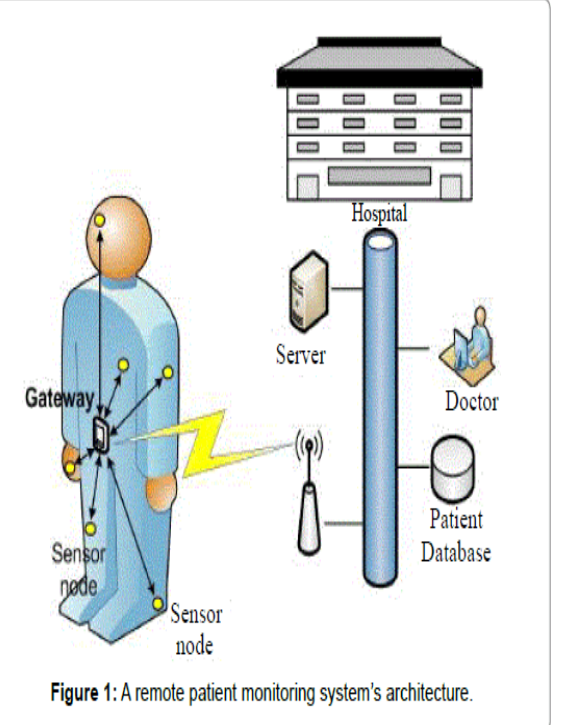

Fig. 3: Remote patient monitoring system

\subsection{Research Issues in Military Applications}

Sensor network plays a vital role in military command line control, figuring intelligence, ccommunications, surveillance, and indicating systems. WSN disposed of attacks in the battlefield where either the data can be corrupted and can send it to the wrong way or people can be attacked by corrupting devices. Sensor network provides security and leading large amount of energy consomptions and finally exit on the node. A sensor movement scheduling algorithm was developed for detection in mobility system, it is very effective and provide extensive simulations. Sensor network has found in very acute application such as object detection and object tracking. These applications require high detection possibility, due to its low and false alarm rate along with bounded detection delay. Sensor networks based on low rate, and nonrefundable sensor nodes, ruin of some nodes by aggressively activities that do not disturb the military tasks due to this its consider is the best approach to use sensor network in the battlefield. Some more applications of the military in a wireless sensor network are monitored. These applications and their working are mentioned in Table 2.

Table 2. Applications and their performance

\begin{tabular}{|l|l|}
\hline Applications & Performances \\
\hline $\begin{array}{l}\text { Monitoring } \\
\text { friendly forces } \\
\text { equipment and }\end{array}$ & $\begin{array}{l}\text { Commanders and leader continuity } \\
\text { monitor the troops and equipment } \\
\text { availability, in the battlefield that is } \\
\text { attached by the sensor network. All } \\
\text { information is collected with a small } \\
\text { sensor and send it to the leader and then } \\
\text { this report are forward it to the upper } \\
\text { level or senior officer for further action. }\end{array}$ \\
\hline $\begin{array}{l}\text { Targeting } \\
\text { valuation }\end{array}$ & $\begin{array}{l}\text { Sensor networks could be merged into } \\
\text { guidance systems. }\end{array}$ \\
\hline $\begin{array}{l}\text { Nuclear } \\
\text { Biological } \\
\text { Attack Detection }\end{array}$ & $\begin{array}{l}\text { sensor network could be organized in } \\
\text { arget area no matter its after and } \\
\text { before it gather battle amage } \\
\text { assessment }\end{array}$ \\
\hline $\begin{array}{l}\text { Timely and accurate detection is very } \\
\text { necessary for nuclear and biological } \\
\text { attacks. Sensor network is used as a } \\
\text { warning system for reaction time, which } \\
\text { drops victims drastically. }\end{array}$ \\
\hline $\begin{array}{l}\text { In Critical environments, routes, paths } \\
\text { could be quickly covered with } \\
\text { wireless sensor networks and it can } \\
\text { thoroughly watch activities that are } \\
\text { going to be opposite forces. Is there any } \\
\text { plan are developed then new sensor } \\
\text { networks should be installed } \\
\text { immediately in battlefield } \\
\text { reconnaissance. }\end{array}$ \\
\hline
\end{tabular}

\subsection{Research Issues in Commercial Applications}

Commercial applications in sensor network include monitoring product quality; event determines, structural health monitoring systems, constructing smart office spaces, vehicular monitoring, Smart Parking, environment control in buildings, cultural property protection, detecting and observing vehicle like car thefts, Organization list control etc. 


\subsubsection{Smart Parking}

Sensor network provides efficient parking system [11] with less expensive investments in new installations and make cheap sensor node which can be used to track the vehicles effectively.it makes effective usage of existing parking by using of sensor nodes network. Parking application uses magnetometers and video cameras but some factors like environmental factors can affect the detection of magnetometers are not accurate and also a video camera which is expensive and not possible to transfer a large amount of data in a wireless environment through multiple hops. One more factor that can affect the video cameras and magnetometers that are a parking lot. S.Lee, D.Yoon and A. Ghosh presented the magnetic sensors along with ultrasonic sensors together for detection of the vehicles in parking system. Smart Parking management system has been developed for monitors the remote parking system and also developed a mechanism for parking reservation system.

\subsubsection{Smart environment}

The smart environment designed to show factors one is human-centered and another is technology-centered. The smart environment has been adapting to complete the needs of end users in term of input, output abilities and human addressed, smart environment design technology-centered, networking solutions, new hardware technologies, and middleware services [12].

\subsubsection{Environmental control in buildings}

Wireless sensor network provides the distributed systems [13] by controlling the temperature inside the office building's room so that the temperature is controlled and cannot disturb the environment, can reduce the energy consumption [14].

\subsubsection{Security for Intra Car}

Sensor network provides security for intra car [15] to achieve fuel efficiency and reduction in the weight automotive, by replacing wired sensor and their cable with the help of sensor network, but still, the security issue is occur due to inherent vulnerability of the wireless platform such as replacement, and highly questionable.

\subsubsection{Detection of event}

Tracking is the most common event of wireless sensor network. A fully distributed protocol has been presented for Collaborative Event Detection and for like (CollECT) [16].

\subsubsection{Interactions of museums}

In future, some objects will introduce to connect with museums, and these articles will be able to give respond with touch and articulation. There is also the possibility to learn and science environment by participating in real-time system and affect the experiments through objects. San Francisco Exploratorium contains the features of data measurements and can affect the experiments for making the availability of paging and confined in the museum.

\subsubsection{Organization inventory control}

Organization contain different types of items and attached those items with sensor nodes so the user can find out the item's location by using the item's number with similar type, and also user can insert new records so, the user should try to attach appropriate sensor nodes with inventories during insertions. User can easily track the exact location of all inventories at the same time.

\subsubsection{Vehicles tracking and detection}

Tracking and detection are different terms. Tracking can determine locally within clusters and then forward it to the base station, while in detection raw data are collected by sensor nodes for determining location of the vehicle and then forwarded to the base station.

\subsection{Research Issues in Environments Applications}

Environmental applications of sensor network include tracking, monitoring environmental conditions that affect the movement of birds, crops and livestock, animals, and insects. It also works in biological detection; precision agriculture, Earth, fire determine; flood determine; biological -complexity with mapping of an environment, the study of pollution, meteorological or geophysical research; planetary exploration etc.

\subsubsection{Biological complexity with mapping of the environment}

For integrated information across progressive spatial scales, biological complexity mapping requires sophisticated approaches. Two technologies like the remote with sensing and automation of the data collection provide the advantages like spatial, shadowy, and temporal resolution at a geometrically declined rate per unit area beside with fees. Sensor network nodes can control, monitor biological complexity of environment by creating connection within Internet.

\subsubsection{Detection of firewood}

Sensor network nodes may intentionally, casually, deployed for the forest, and it may transmit the exact source of fire to users before fire spread and overwhelming. Sensor network node organized by applying radio frequencies and visual system. Sensor network also, prepared by real power methods which contain stellar cells, because for months and even years the sensors may be left unattended. Sensor nodes work corporately and reduce obstacles.

\subsubsection{Floods detection}

Alert system deployed in the US for flood detection. ALERT system developed several types of sensor like rainfall, and weather sensor. These sensors using a pre-defined way to provide information to the rationalized database system. Some sensor nodes supply snapshot and large running queries, along with a small number of a research project like a cougar and device Database Project at the Cornell University.

\subsubsection{Precision Agriculture}

Some level can be monitored to insecticides these level include drinking water, soil abrasion, and high-level air pollution in a real-time system. These levels are monitors to achieve benefits.

\section{SENSORS ATTRIBUTES AND GENERATIONS}

In Table 3. Sensor attributes and generation are presented

Table 3. Attributes and generations

\begin{tabular}{|l|l|}
\hline Attributes & Generations \\
\hline Sensor & Manufacturer \\
\hline Sensing entity of interest & Size \\
\hline communications & Weight \\
\hline
\end{tabular}




\begin{tabular}{|l|l|}
\hline Operating environment & Node architecture \\
\hline Processing architecture & Topology \\
\hline
\end{tabular}

\section{HARD PROBLEMS AND TEHNICAL CHALLANGES}

Sensor network contains common and a number of technical problems for sensor management that show in data processing, and communication system. Due to its severe, uncertain, cruel and dynamic background, it needs to be applying sensor network's constraints like energy and bandwidth, in an ad-hoc network. The sensor network can control and route through common information processing, inquire, and tasking.

\subsection{Ad -Hoc Network's Innovation}

Sensor nodes must have knowledge about the network for properly operate. For support the neighbors with processing and collaboration, each node must know about identity and location of its neighbors. If one sensor fails or new sensor are installed then sensors in ad hoc networks, topology must be created in real time system, also update their information occasionally. Mobile network contains Sensor nodes with their location. Relative positioning algorithms need to be providing when GPS location is not realistic and more expensive.

\subsection{Sensor Networks Routing Control}

Sensor network must trace resources like energy, bandwidth, along with processing power and must be the part of the system to operate independently, change configuration according to requirement and also change dynamically. According to algorithms and software requirements, connections emerge in ad hoc networks. Communication and energy must be well managed by using RF transmission degrade that provide distances which is faster than in free space.

\subsection{Collaboration of Signal's Information \\ Processing}

Sensor network nodes in the ad-hoc network are used to collect data and process that data for the production of useful information. This is a latest area of research and parallel distributed information of union. Combined information is sharing with other nodes consider as important technical concerns that include the amount of information sharing among the nodes and tell us how nodes fuse that information from other nodes. Data Processing of sensor commonly gives an enhanced performance but it also needs more communication's resource like energy etc. Info that creates the communication required more bandwidth at the lower level otherwise it will lose. Fusion approaches use the model-based techniques that range and picking the simple rules to get best results. Sensor node must determine the result with increasing accuracy, and processes should be completed when entire accuracy gained.

\subsection{Task and Query}

The data base is considered a sensor field and used in distributed system sensor used its unique features. Data is needed to be developed for the environment. So the data should be distributed between nodes, and geographically disseminated and connected these nodes with unreliable links. So this is not reliable in military applications where the requirements like low latency rate, and high consistency are to be needed and must fulfill for the battlefield. It is necessary for end users should have a small edge and must be connected to the system to perform task and query [17]. There is a challenge

to develop the language for performing functions in querying and tasking programs, for users so that the database can run queries. Another challenge which is included for efficient distributed mechanisms to run the query, task compilation, and location. One software is human interface network that is hand held unit to developed and accept any type of speech input so the user must have to command on the query to run the function. Sensor network techniques provide survivable, low latency, and security within the network.

\section{CONCULUTION}

Sensor network involves small sensing nodes, computation, and wireless communications skills. Sensors network also consists of routing protocol which depends on different architecture and networks on. Sensor network as considering in the ad-hoc network and mobile network is different according to it challenges they work different according to network and design, sensor also provides the solution in this problem consists on location, deploy, and tracking, are core issues. Coverage tells about the quality of the service like provide information about the surveillances. Sensor network in energy system it converts it into electrical energy. Sensor network does not provide security in an ad-hoc network, malicious code and threats can attack in that

network. Animations of fault tolerance for wireless sensor network topologies become tradeoff and also include network's weakness to multiple fears. Even though the advantages of sensor networks are speedily recognized, but there are some limitations of their applications in large military system. Advance technologies in past phase have been completely changed the situation. One of the best has been present that is MEMS technology, and it is most dependable wireless communication, with low-cost industrialization that produced results inexpensive, and prevailing sensor networking experiences. This type of sensor networks could be useful for new applications and range of environment examined to industrial area instead of outmoded military use. In fact, sensor networks applications are limited in our imaginations, and also considere as a small possibly microscopic network. Anyone can perform automatic continues, and separate monitoring, and also radically improve our knowledge and help to understand the physical atmosphere.

\section{REFERENCES}

[1] I. Howitt, J.A. Gutierrez, "IEEE 802.15.4 Low RateWireless Personal Area Network Coexistence Issues," in Proceeding of Wireless Communication and Networking International Conference, IEEE, pp. 14811486, 2003

[2] J.A. Gutierrez, M. Naeve, E. Callaway, M. Bourgeois, V. Mitter, B. Heile, "IEEE 802.15.4:2001. A Developing Standard for Low-Power Low- Cost Wireless Personal Area Networks, “IEEE Network, pp. 12-19.

[3] Eugene Shih, Amit Sinha, Alice Wang, Anantha Chandrakasan, Rex Min, Manish Bhardwaj 
et.al, Energy-Centric Enabling Technologies for Wireless Sensor Networks, IEEE Wireless Communication, vol. 9, no. 4, pp. 28-39, 2002.

[4] Ian F. Akyildiz, Weilian Su, Yogesh Sankarasubramaniam, Erdal Cayirci;august 2002. A Survey on Sensor Networks, in IEEE Communications Magazine, pp. 102-114.

[5] Sukhwinder Sharma, Rakesh Kumar Bansal, Savina Bansal, "Issues and Challenges in Wireless Sensor Networks," in International Conference on Machine Intelligence Research and Advancement, 2013

[6] A. Flemmini, P. Ferrari, D. Marioli, E. Sisinni, and A. Taroni, Wired and wireless sensor networks for industrial applications," in Microelectronics Journal, vol. 40, pp. 1322-1336, September 2009.

[7] Q. Wang, S. Zhang, Y. Yang, and L. Tang," The application of wireless sensor networks in the coal mine," in $7^{\text {th }}$ International Conference on Information, Communication and Signal Processing, ICICS, 2009

[8] Z. Ke, L. Yang, X. Wang, and S. Heejong," The application of a wireless sensor network design based on ZigBee in petrochemical industry field," in $1^{\text {st }}$ International Conference on Intelligent Networks and Intelligent Systems, pp. 284-287.

[9] R. A. Rashid, S. H. S. Arifin, M. R. A. Rahim, M. A. Sarijari, and N. H. Mahalin, "Home healthcare via wirelessbiomedical sensor network," in IEEE
International on RF and Microwave, pp. 511-514, RFM 2008

[10] S. Lee, D. Yoon, and A. Ghosh," Intelligent parking lot application using wireless sensor networks," in International Conference on Collaboration, Technologies and systems, pp. 48-57, 2008

[11] D. J. Cook and S. K. Das , "Smart environments: technologies, protocols, and applications, New York: John Wiley, pp. 13-15, 2004

[12] C.Y. Chong, F. Zhao, S. Mori, and S.Kumar," Distributed tracking in wireless ad hoc sensor networks," in $6^{\text {th }}$ International Conference on Information Fusion, pp. 431-438, 2003

[13] Tarik Arici, Yucel Altunbasak, "Adaptive Sensing for Environment Monitoring using Wireless Sensor Networks," in Wireless Communication and Networking Conference, IEEE, 2004.

[14] H. Lee, H. M. Tsai, and O. K. Tonguz, ,"On the security of intra-car wireless sensor networks," in $70^{\text {th }}$ Conference on Vehicular Technology, pp.1-5, 2009.

[15] K. P. Shih, S. S. Wang, H. C. Chen, and P. H. Yang, 2008. "CollECT: Collaborative Event detection and tracking in wireless heterogeneous sensor networks," in $11^{\text {th }}$ IEEE Symposium on Computer and Communications, vol. 31, pp. 3124-3126, September .

[16] Y. Yao and J. E. Gehrke," Query processing in sensors Networks," in 1st Biennial Conference on Innovative Data Systems Research (CIDR), 2003. 